\title{
émulations
}

\section{La mise en forme bureaucratique de l'action associative en Tunisie}

\section{Les instruments et l'organisation du travail caritatif à Sfax}

Damiano De Facci

Émulations - Revue de sciences sociales, 2021, $\mathrm{n}^{\circ} 37$, «Associations et bureaucratisation : perspectives africaines ».

\section{Article disponible à l'adresse suivante}

https://ojs.uclouvain.be/index.php/emulations/article/view/defacci

\section{Pour citer cet article}

Damiano De Facci, « La mise en forme bureaucratique de l'action associative en Tunisie. Les instruments et l'organisation du travail caritatif à Sfax », Émulations, n 37 , Mise en ligne le 19 mars 2021.

DOI : 10.14428/emulations.037.03

Distribution électronique : Université catholique de Louvain (Belgique) : ojs.uclouvain.be

(C) Cet article est mis à disposition selon les termes de la Licence Creative Commons Attribution, Pas d'Utilisation Commerciale 4.0 International. http://creativecommons.org/licenses/by-nc/4.0/

Éditeur : Émulations - Revue de sciences sociales / Presses universitaires de Louvain https://ojs.uclouvain.be/index.php/emulations

ISSN électronique : 1784-5734

PUL PRESSES

UNIVERSITAIRES

DE LOUVAIN 


\title{
La mise en forme bureaucratique de l'action associative en Tunisie
}

\author{
Les instruments et l'organisation \\ du travail caritatif à Sfax
}

\begin{abstract}
Damiano De Facci ${ }^{1}$
[Résumé] À travers les cas de deux associations caritatives de la ville de Sfax, en Tunisie, l'analyse se focalise sur trois instruments d'action - le livre comptable, l'organigramme et la base des données des bénéficiaires -, afin de mettre au jour les tensions et les ambiguïtés des dynamiques de mise en forme bureaucratique de l'action associative. Ces tensions et ambiguités montrent que la définition des instruments d'action associative ouvre davantage de marges de manœuvre pour les responsables des associations. Dans ce cadre, la conformité des procédures de l'association aux normes imposées par l'État, qui lui assure la légitimité de son action, semble moins le résultat d'une réelle volonté de collaboration avec l'État qu'une appropriation des logiques étatistes de la part des responsables des associations. Ces responsables jouent un rôle grandissant, en devenant à la fois des managers de la charité et les garants du fonctionnement de la chaîne sectorielle. La mise en forme bureaucratique de l'action associative se révèle ainsi être un processus de passage des associations de la fonction d'intermédiation idéologique et clientéliste à une forme de gouvernement personnalisée, fondée sur les responsables-managers des associations.

Mots-clés : associations, Tunisie, instruments d'action publique, bienfaisance, bureaucratie

The bureaucratization of organisational action in Tunisia. The instruments and the organisation of charity work in Sfax

[Abstract] The analysis stems from the case of two charitable associations in the city of Sfax, Tunisia, and focuses on three instruments - accounting books, organisational charts, and databases of beneficiaries - to highlight the tensions and ambiguities within the bureaucratic form of organisational action. Such tensions and ambiguities demonstrate how the instruments of organisational action increase the margins of manoeuvre of charities' managers. In such a context, the adherence of organisational procedures to State norms, and thus the legitimacy of these organisations, rests on the appropriation of State logics by charities' managers, rather than on a real will to collaborate with the State. These managers come to play an increasingly central role, becoming at the same time the managers of their charities and the guarantors of the whole charity sector. The bureaucratic form of organisational action thus reveals a shift in the role of organisations, from serving the function of ideological mediation and the mediation of clienteles, to embodying a form of personalised government, based on the managers-guarantors of organisations.
\end{abstract}

Keywords: civic associations, Tunisia, public action instruments, charity, bureaucracy

La révolution tunisienne et la chute du régime de Ben Ali le 14 janvier 2011 ont été suivies par un essor des associations. Entre 2011 et 2013, des milliers d'associations

\footnotetext{
${ }^{1}$ CESSMA, Paris.
} 
sont créées $^{2}$ dans la foulée des mouvements sociaux de la révolution et dans le double contexte du soutien institutionnel et économique international à la transition démocratique, et de l'organisation du pluralisme politique. Dans ce cadre, une multiplicité d'acteurs associatifs, privés, étatiques et internationaux participent à la mise en forme de l'action associative à travers l'élaboration de normes et de procédures qui assurent sa légitimité et son financement.

En opposition aux normes en vigueur sous le régime autoritaire, l'enjeu des processus de bureaucratisation (Bayart, 2013 ; Hibou, 2013) définissant le statut, le cadre d'action et de financement des associations, prônés notamment par l'État et par les acteurs internationaux est la création de normes pour une action associative démocratique. Grâce aux formations assurées par les bureaux de l'État ${ }^{3}$, par les organisations internationales, ou par des consultants privés, les associations « absorbent » les recommandations et les principes classiques de la "société civile internationale » (Ferré, 2018 ; Sigillò, 2016), et formulent des discours critiques concernant leur propre action.

Le plus souvent, les activistes associatifs rencontrés affirment que les associations doivent garder une neutralité par rapport à l'État et aux partis politiques : voilà le premier principe auquel celles-ci devraient se conformer. La conscience de leur propre rôle passe également au travers de la critique des pratiques d'avant 2011. Le discours d'une agente de terrain de l'UTSS (Union tunisienne de solidarité sociale), ancienne structure de distribution d'aides et de développement qui essaie de se renouveler après le 14 janvier 2011, peut servir d'exemple à ce propos.

Avant la révolution, on était toujours lié à la politique de l'État, maintenant on peut choisir les domaines d'intervention. Avant il y avait un certain pilotage ; plus ou moins, hein ! Mais aujourd'hui on se sent plus libre. [...] C'était toujours les autorités locales qui intervenaient pour proposer les noms des bénéficiaires : elles nous donnaient les listes et on se retrouvait obligé d'accepter ça, on ne pouvait pas dire non. Maintenant on peut dire non. (Entretien avec Aïda, Kasserine 20/05/14) .

Le discours accompagnant la bienfaisance est mis en contraste avec les pratiques clientélistes des autorités publiques, et la liberté des associations est attribuée à la fin de la violence illégitime du régime autoritaire. Au-delà d'une simple opposition entre nouvelles et anciennes associations - liberté contre autoritarisme -, la rupture temporelle distingue surtout la légitimité des pratiques des associations.

En se focalisant sur les instruments d'action associative, soit sur les dispositifs qui en structurent les programmes en composant des logiques d'action et des conceptions du rapport à l'État multiples, le présent article traitera des processus de bureaucratisation

\footnotetext{
${ }^{2}$ Au cours de l'année 2011, la Tunisie enregistre la création de plus de 2000 nouvelles associations, alors qu'elle en comptait seulement 9600 en 2010 ; l'année 2012 voit la création de près de 3000 associations (FFF, 2013).

${ }^{3}$ Notamment par le Centre d'information, de formation, d'études et de documentation sur les associations (IFEDA), créé en 2000 et placé sous la tutelle du Premier ministère.

${ }^{4}$ Pour une analyse politique du rôle de I'UTSS sous le régime de Ben Ali, voir les travaux de Béatrice Hibou (Hibou, 2006).
} 
des associations du point de vue de leur quête de légitimation et de distinction par rapport aux pratiques autoritaires. À partir de cas empiriques issus d'un travail de terrain ethnographique ${ }^{5}$ réalisé entre 2014 et 2017 dans la ville de Sfax en Tunisie, l'article se focalisera sur les ressorts et les tensions résultant de l'organisation bureaucratique du travail caritatif dans le cas d'un réseau d'associations qui essaient de coordonner leur action. Après avoir, dans une première partie, situé historiquement l'essor des associations tunisiennes et avoir précisé le concept d’instruments de l'action associative, la deuxième partie abordera notamment l'analyse de deux trajectoires d'associations caritatives de Sfax. L'analyse mettra en exergue le rôle de certains instruments d'action définis par les associations - comme le livre comptable, l'organigramme, la base des données des bénéficiaires - et les marges de manœuvre que ces instruments donnent aux acteurs associatifs. La troisième partie sera consacrée à une discussion des processus de bureaucratisation et de l'articulation entre gouvernement technique, gouvernement partisan et gouvernement personnel que l'analyse des instruments d'action associative révèle.

\section{Entre libéralisation et démocratisation : de l'association- instrument aux instruments de l'action associative}

L'essor des associations entre 2011 et 2013 a été soutenu par la politique de l'État en matière d'emploi, et notamment par le programme de Service civil volontaire (SCV) ${ }^{6}$ de l'ANETI', qui prolonge le Fonds national de l'emploi 21.21 institué en 1999 pour l'insertion des demandeurs d'emploi. Suite à la révolution, le ministère de l'Intérieur commence à donner les autorisations très vite aux nouvelles associations. La promulgation en septembre 2011 de la nouvelle loi ${ }^{8}$ régissant les associations simplifie les procédures administratives et rend effectif le régime de déclaration au Premier ministère, en mettant les associations à l'abri du contrôle du ministère de l'Intérieur. Par ailleurs, la nouvelle Constitution de janvier 2014 adopte des dispositions qui consacrent le principe de subsidiarité, la communalisation de l'ensemble du territoire, la généralisation des élections dans les collectivités locales et la participation citoyenne aux décisions locales.

Dans ce cadre, la participation des associations à l'action publique devient un enjeu de réforme de l'État dans un sens démocratique (PNUD, 2015). Néanmoins, elle n'est pas un fait de la révolution : elle a un double ancrage historique. Les premières ouvertures

\footnotetext{
${ }^{5}$ Ce terrain faisait partie d'une plus grande enquête empirique, menée entre fin 2013 et mi-2017, en vue de la préparation d'une thèse de doctorat en sociologie sur la participation des associations à l'action publique en Tunisie postrévolutionnaire, et soutenue en 2019. L'ethnographie a consisté en de longs séjours sur place, des observations systématiques des activités associatives et des entretiens approfondis avec les responsables et les activistes des associations. Une partie des entretiens analysés dans cet article a été menée en équipe avec Fabio Merone et Ester Sigillò. ${ }^{6}$ Il s'agit d'un stage en association destiné aux diplômés du supérieur primo-demandeurs d'emploi, avec une indemnité de 200 DT et la sécurité sociale.

${ }^{7}$ L'Agence nationale pour l'emploi et le travail indépendant (ANETI) est un établissement placé sous la tutelle du ministère de la Formation professionnelle et de l'Emploi.

${ }^{8}$ Loi 88-2011 du 24 septembre 2011.
} 
libérales du régime de Ben Ali, entre 1987 et 1992, ont entraîné pour la première fois une poussée du nombre des associations, dans le contexte plus large des transformations socioéconomiques entamées à partir de la deuxième moitié des années 1980 avec l'ajustement structurel. À partir des années 2000 notamment, les politiques publiques s'appuient de plus en plus sur des acteurs associatifs pour mener des actions sociales et de développement de proximité (Allal, 2016 ; Ben Amor, 1994 ; Laroussi, 2009). En effet, les réformes successives au Programme d'ajustement structurel de 1987 avaient produit des effets imprévus en affectant le niveau de vie des catégories sociales à faibles revenus et, de manière plus générale, en ébranlant les structures de protection sociale. Cela a incité l'État à construire un discours politique très ouvert aux exigences internationales de « bonne gouvernance » et d'appui à la société civile (Laroussi, 2009). Néanmoins, la quasi-totalité 9 de ces associations faisait partie du dispositif autoritaire de contrôle sur la population, ainsi que de l'appareil de légitimation du régime (Ben Achour, 2011). Elles étaient à la fois encadrées par le régime, inféodées par le parti au pouvoir, ou encerclées par les pressions judiciaires et policières, et empêchées d'avoir accès aux populations auxquelles elles s'adressaient (Ben Achour, 2011 ; Redissi, 2007). En revanche, le boom associatif des années 2011 et 2012 relève des transformations politiques qui ont suivi le 14 janvier 2011. Il se situe à la fois dans la rupture du 14 janvier - avec l'appropriation de l'héritage des revendications des mouvements de révolte - et dans la continuité des transformations néolibérales de l'État et de l'action publique.

Instruments des politiques publiques libéralisées depuis les années 1980, notamment dans le champ de l'emploi (De Facci, 2016 ; Hély, 2009; Simonet, 2010), les associations doivent désormais participer à l'action publique en tant qu'acteurs de démocratisation (De Facci, Som-1, 2017; Laville, Salmon, 2015). Dans ce cadre, la définition d'instruments d'action publique (Halpern, Lascoumes, Le Galès, 2014 ; Lascoumes, Le Galès, 2004) de la part des associations permet d'analyser l'essor des associations tunisiennes non seulement par le haut des politiques gouvernementales, mais aussi par le bas des registres d'action des acteurs associatifs, de leur expérience et de leurs pratiques (Lefèvre, 2008). En particulier, l'entrée par les instruments permet d'analyser les processus de bureaucratisation associative par les jeux des acteurs qui participent à la construction des instruments, et par les effets du travail des instruments sur l'action associative et sur la reconfiguration de l'État. Cette analyse reprend la définition d'instrument d'action publique donnée par Pierre Lascoumes et Patrick Le Galès : « un dispositif technique à vocation générique porteur d'une conception concrète du rapport politique/société et soutenu par une conception de la régulation »(Lascoumes, Le Galès, 2004 : 14). Néanmoins, son usage dans le cadre de l'action associative veut aller au-delà de l'analyse de l'association-instrument. Parler d'instruments d'action associative signifie non seulement se décentrer de l'analyse des objectifs des politiques publiques en mettant l'accent sur les dispositifs qui en structurent les programmes, mais aussi de réfléchir sur la multiplicité d'acteurs qui définissent les instruments d'action publique, sur les

${ }_{9}^{9}$ À l'exception notamment de cas remarquables comme la LTDH, I'ATFD, I'AFTURD, le CNLT, Amnesty International. 
relations entre associations et État et sur les formalités qui font exister l'association en tant qu'acteur collectif. À partir de ce cadre analytique, le cas de deux associations tunisiennes permettra de mettre en lumière les tensions et les ambiguïtés qui émergent autour de la mise en place des instruments du travail caritatif.

\section{Deux trajectoires d'associations à Sfax}

La configuration d'actions associatives étudiée dans le présent article se situe au premier abord à l'intersection de l'activisme politique et des pratiques religieuses. L'analyse se focalisera en particulier sur deux associations de Sfax faisant partie d'un réseau d'associations à référent islamique « La main dans la main ${ }^{10}$ », aux activités relativement hétérogènes, mais plutôt centrées sur la charité religieuse et la prédication. La plupart des membres fondateurs et des activistes de ces associations sont issus du parti islamiste Ennahdha (Merone, Soli, 2013 ; Sigillò, 2020a). Parti d'opposition aux régimes de Bourguiba et Ben Ali, beaucoup de ses militants ont subi la répression, la prison et l'exil. Légalisé après la révolution, Ennahdha gagne les élections de 2011 et participe à tous les gouvernements qui se sont succédé depuis 2012.

L'association « La source de la charité de Sfax » naît début 2012. Son président et fondateur est Habib, un ancien militant d'Ennahdha. Habib commence à militer au sein du parti pendant les années 1980. À partir de 1999, il dirige la cellule des « affaires sociales » d'Ennahdha pour l'ensemble du gouvernorat de Sfax. Il s'agissait de s'occuper des familles des prisonniers et des anciens prisonniers politiques du mouvement islamiste. Le groupe est composé par quelques militants qui ont un réseau de contacts dans l'ensemble du gouvernorat, récoltent l'argent et le redistribuent : tout le travail est mené en cachette, dans une cellule isolée et seulement par transfert monétaire. Après la révolution, ce noyau constitué par la cellule des affaires sociales d'Ennahdha de Sfax se transforme en association - les membres de la cellule font partie du bureau de l'association -, en changeant sa raison d'être : il s'agit d'aider tout le monde, et non seulement les camarades. L'aide consiste désormais en biens alimentaires, équipements et vêtements. Habib établit une continuité forte entre son expérience passée et son association :

L'association existait déjà en pratique, mais on a eu l'acceptation officielle seulement fin 2011 début 2012. Avant elle n'était pas formalisée, on travaillait en cachette. Mais la structure était déjà là. Maintenant on peut travailler librement. [...] Aujourd'hui je préfère l'associatif au politique, parce que dans le travail politique il y a des limites, il faut faire attention ; avec l'association je peux travailler avec tout le monde. Pour nous c'est l'aide pour l'aide, ce n'est pas pour le gain, c'est une valeur religieuse, je veux aider toute personne nécessiteuse. On arrive à récupérer pas mal d'argent, les gens me connaissent, parfois ils m'appellent pour donner de l'argent, ils savent que je ne le fais pas pour moi, que j'aide les gens. (Entretien avec Habib, Sfax 29/04/15)

\footnotetext{
${ }^{10}$ Les noms des acteurs et des associations enquêtés ont été anonymisés.
} 
L'expérience du travail en clandestinité est très importante, puisque Habib et ses camarades ont pu connaître les familles des prisonniers politiques et les prisonniers politiques eux-mêmes, et ont pu constituer un réseau, en sollicitant parfois des hommes d'affaires.

Le changement de la situation politique du pays permet de formaliser l'association et de travailler pour atteindre " tout le monde », sans sélection a priori. Il représente une bifurcation, dans laquelle Habib affirme avoir choisi l'associatif. Selon l'explication fournie, le politique implique des limitations au travail associatif et à l'universalité de l'aide : si, avant 2011, il s'agissait d'un horizon obligé, la chute du régime représente une opportunité de choix. D’ailleurs, ce choix de détachement du politique est lié à la spécialisation dans le travail d'aide, surtout dans son côté le plus organisationnel :

On était doués pour ce genre de travail et on avait une véritable connaissance des réseaux. C'est pour cela que notre association travaille mieux que les autres associations, mais en même temps c'est complètement différent. Il s'agit d'aider tout le monde, mais on arrive à aider beaucoup moins de personnes. Avant, il y avait 8000 prisonniers politiques à Sfax : on arrivait à aider la plupart des familles. Aujourd'hui, la logique est différente. (Entretien avec Habib, Sfax 25/05/17)

L'« Association de bienfaisance de Sfax », quant à elle, est fondée en 2011 sous le nom de Rahma, la "miséricorde ", nom à forte connotation religieuse (Ben Néfissa, 1992).Son histoire est liée à celle de Skander, directeur général de l'association depuis 2013. Ancien militant d'Ennahdha, Skander part en Allemagne en tant que réfugié politique dans les années 1980. Il fait des études professionnelles pour le travail social et commence à travailler à la Diaconie, la structure caritative de l'Église protestante allemande, comme assistant de direction à Duisburg. Dans ce cadre, il a l'opportunité de participer à la gestion des répercussions de la fermeture de la grande usine de la Thyssen :

Il fallait gérer la fracture sociale, puisque la fermeture de l'usine avait provoqué des millions des chômeurs dans le bassin sidérurgique. Pour l'encadrement et la gestion sociale de la crise, il y a eu l'alliance entre l'Église, l'État et les partis politiques. (Entretien avec Skander, Sfax 20/01/16)

Par la suite, il travaille pour une fondation syndicale proche du parti social-démocrate allemand et pour l'Organisation internationale des migrations (OIM) à Düsseldorf, Hambourg et Berlin. Après la révolution, il intègre l'équipe de l'OIM en Tunisie et s'occupe du développement de la politique de l'immigration. Il revient à Sfax en 2013 et commence un travail de réorganisation de Rahma. Son expérience dans les organisations caritatives protestantes et sa proximité avec le mouvement islamique tunisien lui donnent un modèle crédible et la légitimité pour le proposer. En effet, ses missions sont le changement de nom et de structure de l'association : l'association devient ainsi " Association de bienfaisance de Sfax » et son chantier prioritaire devient le soutien aux orphelins. Par la suite, le travail de Skander se concentre surtout sur le marketing de l'organisation : 
Il faut définir des stratégies de communication, les produits, les clients. Contrôle en input et output : en input par une convention avec le donateur; en output par un compte rendu de la comptabilité. On stipule une convention avec les donateurs, le 12,5\% est la partie qui reste à l'association, selon le droit religieux. (Entretien avec Skander, Sfax 20/01/16)

Sa réorganisation de l'association part donc du langage et des dispositifs de financement et vise à créer la confiance autour de l'association par les compétences dans la gestion des projets.

Les deux associations ont des dimensions comparables : les deux comportent en 2016 environ une centaine d'adhérents. Néanmoins, l'« Association de bienfaisance de Sfax » est beaucoup plus structurée par rapport à " La source de la charité de Sfax », qui ne compte qu'une assistante sociale salariée : l'« Association de bienfaisance de Sfax » a un directeur général et un directeur exécutif salariés, ce qui lui permet d'organiser le travail de manière professionnelle et de gérer davantage de projets. Les deux associations sont liées à l'Organisation tunisienne de développement social (OTDS) et au Programme d'appui à la société civile (PASC), qui les poussent à diversifier le travail caritatif, en mettant en place des projets d'activités génératrices de revenus.

\subsection{La remise en cause de la légitimité associative : le livre comptable}

Pendant la période de 2012-2013, caractérisée par une conflictualité sociale et politique aiguë, les associations caritatives sont ciblées par une série de polémiques politiques et de scandales journalistiques fondés sur deux accusations principales : d'un côté, elles seraient le relais associatif du parti d'Ennahdha auquel elles assureraient une clientèle, de l'autre, elles viseraient à « islamiser » la société tunisienne, en étant même un véhicule d'idéologies politiques " extrémistes». Les deux accusations ont en commun de pointer du doigt le manque de transparence des financements associatifs. Avec le gouvernement « technocrate » de 2014, les activités de dizaines d'associations de charité et de prédication sont " gelées », contestant l'opacité de leurs comptabilités (Merone, Sigillò, De Facci, 2018).

Tout en mettant l'accent sur le caractère répressif et discrétionnaire des pratiques de l'État envers les associations, les entretiens révèlent un repositionnement progressif par rapport à ces épisodes. C'est le cas de Habib, président de « La source de la charité de Sfax ». Depuis notre premier entretien en 2015, lorsqu'il jugeait que le gel des associations « n'est qu'un acte politique criminel; si c'était mon association, je n'aurais pas arrêté le travail, ils ne peuvent rien faire » (Entretien avec Habib, Sfax 29/04/15), Habib a partiellement changé d'idée. En parlant d'une association qui a été sanctionnée en raison de sa comptabilité opaque, il évoque la façon dont elle « n’a pas bien géré les choses, elle a traîné, et on est arrivé à la limite : tu cumules, tu cumules et ça arrive au bout » (Entretien avec Habib, Sfax 15/05/16). L'affaire n'est plus jugée à l'aune du conflit politique, mais de la dimension critique de la publicité : 
Le problème est qu'on a toujours été habitués à travailler en secret : on a ce problème de confiance même aujourd'hui. On était en clandestinité pour trop de temps et on a pris l'habitude. (Entretien avec Habib, Sfax 15/05/16)

Ce repositionnement est évident également dans les interactions d'enquête. Pendant mon terrain, au début des entretiens, les responsables des associations caritatives me montraient tout de suite le livre comptable, où sont notées toutes les transactions d'argent : les dons, les financements, les dépenses. Cet acte était accompagné par un discours où ils affichaient le souci de transparence, et leur professionnalisme dans la comptabilité de l'association. Ce geste relève de la construction du discours public des associations caritatives face aux campagnes de remise en cause de leur légitimité (De Facci, 2019).

En effet, l'affaire du gel des associations met en cause non seulement l'honnêteté des responsables associatifs, mais aussi leurs compétences. Le livre comptable devient ainsi l'enjeu sur lequel s'attachent à la fois les soupçons des uns et les justifications des autres. À l'épreuve du livre comptable, les associations de charité mobilisent un registre fondé sur les compétences, en le centrant sur la gestion de l'association. La comptabilité oblige les associations à standardiser leurs procédures et à professionnaliser leurs bureaux, en acceptant les limites imposées par le cadre juridique. Ces compétences sont mises en avant pendant les entretiens, comme dans le cas du président de l'« Association de bienfaisance de Sfax » :

Notre association est complètement transparente (procédures claires, papiers en règle, financements transparents) ; la gestion de l'association est strictement contrôlée, nous travaillons de manière professionnelle. Nous sommes en train d'obtenir une certification ISO de standard international, on sera la première association en Tunisie à l'avoir. (Entretien avec Lotfi, Sfax 13/12/14)

Ainsi employée, la logique des compétences devient le registre sur lequel est justifiée la transformation de l'action associative : « La loi doit être au-dessus de tout, certaines associations n'ont pas bien géré leur budget. Notre bilan est journalier, on enregistre tout don reçu, matériel ou financier » (Entretien avec Hédi, Sfax 20/04/15). Le nouveau cadre est opposé aux anciennes pratiques, notamment à la « politisation » des pratiques associatives : « avant on faisait n'importe quoi » (Entretien avec Habib, Sfax 15/05/15); ou encore, comme l'affirme Skander, le directeur général de l'« Association de bienfaisance de Sfax » : «Il faut professionnaliser et centraliser le travail associatif ; surtout il faudrait éviter la politisation des associations, comme c'est le risque avec les petites associations » (Entretien avec Skander, Sfax 20/01/16).

À travers la maîtrise de l'instrument du livre comptable, les deux associations analysées s'approprient les registres de légitimation de la compétence et de la professionnalisation. Néanmoins, ces registres signifient moins l'adéquation à des normes standardisées d'efficacité et d'accountability, de calculabilité et de comparabilité que de réguler des rapports légitimes entre associations et État, tout en laissant des marges de manœuvre aux responsables des associations. L'analyse de l'organigramme du travail caritatif et de la base des données des bénéficiaires montrera les tensions entre le 
maintien de cette marge de manœuvre et la mise en forme de l'action associative selon les logiques de la transparence, de la professionnalisation et de l'efficacité.

\subsection{L'efficacité en question : l'organigramme du travail caritatif}

Au-delà de la légitimation, le problème d'une approche professionnelle des procédures de gestion des associations relève de l'organisation sociale du travail associatif. Un manager associatif comme Skander prône la réforme du travail caritatif, avec la fusion des petites associations et la sectorisation des activités. Cependant, cette tentative de réforme du milieu associatif sfaxien rencontre des formes de résistance, menées par les responsables d'association engagés notamment dans une compréhension plus politique du milieu associatif sfaxien. La scène principale du conflit est le réseau « La main dans la main », qui ne réunit pas seulement les associations caritatives, mais tout un ensemble d'associations à référent islamique, de prédication, et notamment les associations des imams.

L'idée de réunir les associations a pour objectif de rendre le travail associatif plus efficace. « Il y a des obstacles pour les organisations qui restent trop petites : il faut choisir entre une grande institution qui travaille avec l'État et les petites, qui ne peuvent pas débloquer des ressources » (Entretien avec Skander, Sfax 20/01/16).

D’après Skander, dans un contexte de dépendance à l'argent des bailleurs de fonds, les associations devraient apprendre à débloquer des ressources tunisiennes et devenir la «banque d'elles-mêmes ». Pour cela, il faut conquérir la confiance des donateurs et donc montrer la gestion professionnelle de l'organisation. Mais il faut surtout réunir les petites associations en une seule grande association qui puisse à la fois être partenaire de l'État et compter sur un historique de bons résultats de ses projets. La loi sur les fondations religieuses pourrait ainsi aider cette organisation des associations, moins par la réinstitution d'une fondation religieuse de chaque mosquée ${ }^{11}$ que par l'institution d'une fondation civile, c'est-à-dire d'une institution régionale de récolte des dons, " qui pourrait arriver à gérer jusqu’à 50 millions de DT » (Entretien avec Skander, Sfax 20/01/16). L'organisation du travail caritatif ne devrait donc pas être réticulaire comme elle l'est dans le cadre du réseau « La main dans la main », car « il n'est pas possible de travailler avec une organisation horizontale »(Entretien avec Skander, Sfax 20/01/16).

Le renouvellement de l'« Association de bienfaisance de Sfax » est justement pensé pour englober le travail des autres associations du réseau. Néanmoins, le projet de réforme a échoué. L'« Association de bienfaisance de Sfax » est clairement stigmatisée par certains responsables des associations du réseau comme « une association qui a une volonté de domination sur les autres associations » (Entretien avec Habib, Sfax 17/01/16). Les accusations dont souffre l'« Association de bienfaisance de Sfax » arrivent

\footnotetext{
11 II s'agit de l'institution ottomane du habous, abolie à l'indépendance du pays, qui a fait l'objet d'une proposition de loi pour sa réinstitution à l'Assemblée nationale constituante, entre 2012 et 2014, de la part de députés islamistes et d'une coalition d'associations, dont les associations du réseau « La main dans la main » de Sfax.
} 
jusqu'à mettre en cause sa légitimité, en insinuant que certains de ses responsables étaient des membres de l'ancien régime (Entretien avec Habib, Sfax 17/01/16).

Une solution intermédiaire est donc trouvée avec la constitution du « Réseau des associations de développement de Sfax ", entièrement dédié à la charité religieuse, au développement social, et au développement de la ville (Sigillò, 2020b). En partant de l'échec du réseau «La main dans la main », organisé comme pôle idéologique, ce réseau est reconstruit à partir de quelques associations caritatives, donc selon une logique sectorielle. Réunissant six associations de charité, le réseau devrait servir à partager le travail et les contacts, afin d'obtenir plus de crédibilité à la fois aux yeux des autorités publiques et des possibles donateurs.

Les discussions sur l'efficacité de l'action associative mettent en lumière les tensions entre la volonté d'autonomie et la coordination de l'action. Néanmoins, le nouvel organigramme entraîne des transformations de l'action elle-même : les associations se sectorisent, notamment en s'éloignant des activités de prédication et des initiatives des imams, et en recentrant les activités de charité sur les partenaires, les bailleurs de fonds et les bénéficiaires. Comme d'autres associations du réseau caritatif, l'« Association de bienfaisance de Sfax » et « La source de la charité de Sfax » travaillent désormais en collaboration avec les institutions administratives locales, notamment la délégation et la municipalité, dans une logique de subsidiarité. Des acteurs nationaux et internationaux jouent également un rôle important pour le réseau caritatif : de l'Organisation tunisienne de développement social (OTDS) aux organismes de coopération comme le Programme d'appui à la société civile (PASC) ou le PNUD. Au sein de ce nouveau réseau, les activités caritatives sont assimilées à un « secteur » d'activité, tandis que les activités d'éducation visent désormais le plus souvent l'environnement, le recyclage, l'éducation routière, la citoyenneté active et la gouvernance locale participative, plutôt que la prédication religieuse. D’ailleurs, toujours présent comme « valeur», le discours religieux tend à laisser sa place à l'argument du secours aux « pauvres », dans la continuité des politiques sociales et économiques de « sortie de la pauvreté » centrées sur l'individu et sa famille.

\subsection{La bureaucratisation du travail caritatif : la coordination et la base des données}

\subsubsection{La coordination par les procédures}

Les tentatives de collaboration entre associations procèdent au sein des réseaux formalisés et par contact personnel. Elles aboutissent à des mécanismes de coordination du travail caritatif et à des procédures standardisées d'intervention et d'accès aux aides. À la demande d'aide de la part d'un particulier suivent la constitution d'une fiche d'identification, une visite de terrain sans préavis, et l'insertion éventuelle dans une liste d'attente. Ces procédures sont gérées par les membres de l'association ou par 
des assistantes sociales ${ }^{12}$, pour la plupart diplômées et salariées à travers un contrat de Service civique volontaire (SCV). L'aide consiste notamment en biens alimentaires, équipements et vêtements. Il s'agit de dons faits à l'association, souvent sollicités par les responsables auprès de grossistes ou grands vendeurs, ou de produits achetés par l'association, grâce aux dons reçus en argent, ou aux financements par projets des bailleurs de fonds internationaux. Les biens à distribuer sont stockés au siège de l'association et catalogués ; de même, les transactions financières sont enregistrées sur le livre comptable.

Les associations caritatives sfaxiennes essaient de coordonner leur travail à travers l'adoption d'un logiciel commun pour la gestion de la base des données des bénéficiaires, afin de rationaliser le travail et d'éviter le phénomène des doubles aides. Ce logiciel représente un véritable enjeu pour la professionnalisation du travail caritatif : des tentatives différentes sont menées en ce sens, sans qu'on ait dépassé le seuil de la collaboration entre deux ou trois associations. La coordination passe également à travers le transfert des ressources entre associations en cas de besoin et l'utilisation de numéros de téléphone en série. De même, le territoire de la ville est quadrillé : a priori, chaque association travaille dans un secteur particulier. Ce travail de coordination est certainement le résultat d'une volonté de se réunir de la part des responsables des associations, mais il a profité de l'encadrement d'un tissu associatif plus large et du soutien technique d'experts en management des organisations, « internes » comme Skander, ou « externes » comme Fakhri, président d'une association de loisirs des jeunes de Sfax et consultant en stratégie des organisations.

\subsubsection{Construction et usages de la base des données : le cas du couffin de ramadan}

L'activité des associations de charité se focalise surtout sur les quartiers considérés comme défavorisés. L'identification de ces quartiers est fondée sur un récit qui présente la marginalisation d'une manière assez routinière : les « preuves » en sont « la drogue, l'alcool, la prostitution, les viols, des situations familiales très critiques, les maisons mal bâties » (Entretien avec Habib, Sfax 29/04/15). Dans le cadre de cette identification, la « base des données » de l'association est un véritable instrument administratif qui façonne le travail caritatif : elle est à la fois un instrument de triage des profils et une caution de légitimation envers les autorités publiques et les bénéficiaires.

Notre association a une centaine de membres volontaires, pas toujours disponibles, et une salariée, une assistante sociale qui s'occupe de la base des données et des dossiers des familles nécessiteuses. (Entretien avec Habib, Sfax 29/04/15)

Le choix des bénéficiaires est toujours confié aux réseaux personnels et supporté par le travail « des femmes » qui s'occupent de la base des données. Il s'agit des assistantes sociales et des autres personnes - normalement toujours des femmes - qui s'occupent du triage du matériel à distribuer et qui connaissent les familles qui postulent grâce

12 L'usage du féminin correspond à un constat empirique autant qu'à l'énonciation des responsables des associations. 
aux visites de terrain. Cette partie du travail est clairement genrée dans le cas de toutes les associations caritatives analysées, les femmes étant considérées comme plus à l'aise avec le contact humain, l'évaluation du matériel et l'allocation des ressources. Ce sont des femmes qui quadrillent le territoire du secteur où chaque association caritative travaille, pour recueillir l'information afin d'organiser l'intervention, en collectant par exemple le nombre d'orphelins, veuves, divorcées, chômeurs, fils emprisonnés par famille (Entretien avec Fathi, Sfax 04/05/15).

Le travail caritatif se construit de plus en plus comme une intervention bureaucratisée. Cette dynamique de bureaucratisation présente le double mouvement de la mise en place de règles et de standards capables de garantir un traitement égal et impersonnel, d'une part, et du travail personnel de la mise en catégorie et de l'application des critères, d'autre part. Le cas de la distribution du couffin de ramadan (couffin de denrées alimentaires destiné aux familles nécessiteuses pour le mois de ramadan) organisée par « La source de la charité de Sfax » est à cet égard emblématique ${ }^{13}$. La procédure est encore une fois standardisée : le demandeur d'aide prend contact directement avec l'association; par la suite, l'association procède à la visite du ménage, par un comité de contrôle à domicile composé de deux assistantes sociales et deux hommes. Les dossiers des ménages sont insérés dans une base des données et, par la suite, une liste des bénéficiaires est élaborée. En 2017, l'association dispose d'environ 700 couffins : chacun coûte 75-85 DT ${ }^{14}$. « On a eu 20000 DT, moins que les autres années, puisqu'il y a une forte crise économique, mais on a réussi quand même à faire plus de couffins : l'année dernière on en avait environ 500 » (Entretien avec Habib, Sfax 25/05/17).

Le couffin contient des denrées alimentaires de base, comme la farine, la semoule, les pâtes, le lait, l'huile ; chacun reçoit en outre un morceau de viande de poulet. Les associations commencent la distribution du couffin une dizaine de jours avant le début du jeûne : la campagne dure normalement trois ou quatre jours. Au-delà des personnes présentes dans la liste établie par l'association, d'autres noms ont été donnés par le mouatmad ${ }^{15}$. Lorsque quelqu'un qui n'est pas sur la liste arrive au guichet de l'association, son premier acte est souvent la présentation de sa carte de $\operatorname{soin}^{16}:$ de cette manière, il revendique un droit fondé sur une situation reconnue par l'État, ou bien il reconnaît une continuité entre l'association et l'État, dans son caractère d'aide et de sécurité sociale. En revanche, la justification du refus de l'association, qui peut aboutir à la dispute, se fonde sur le fait de la séparation de l'État: les demandeurs d'aide devraient, donc, se diriger vers le omda ${ }^{17}$ ou le mouatmad.

\footnotetext{
13 Les analyses suivantes sont fondées sur les observations menées avec Ester Sigillò lors la deuxième journée de la distribution du couffin de ramadan 2017 au siège de « La source de la charité de Sfax », Sfax 25/05/17, ainsi que sur les entretiens répétés avec le président de cette association et les autres entretiens menés avec les responsables des associations caritatives.

${ }^{14}$ Dinars tunisiens. Entre 2014 et 2017 la valeur du dinar tunisien a varié entre 0,5 et 0,3 euro.

15 II s'agit du responsable de la délégation, une subdivision administrative du gouvernorat.

${ }^{16}$ Il s'agit de la carte des bénéficiaires de la Caisse nationale d'assurance maladie (CNAM).

17 II s'agit du responsable du secteur, une subdivision administrative de la délégation.
} 
«Nous ne sommes pas l'État, nous distribuons aux pauvres et aux nécessiteux, nous ne reconnaissons ni gouverneur ni délégué ni rien » (Entretien avec Habib, Sfax 25/05/17). Habib se rend compte que beaucoup de gens en auraient véritablement besoin, mais il réaffirme que : "l'association a ses limites : déjà il y a une liste de 1500 personnes à partir de la base des données de l'association, tandis qu'on a l'argent seulement pour en aider 700. Cependant, certaines personnes prétendent et ne comprennent pas les explications » (Entretien avec Habib, Sfax 25/05/17).

Néanmoins, au-delà du conflit, les responsables de l'association écoutent les histoires des demandeurs et se consultent entre eux pour décider que faire des situations non incluses dans la liste. Une liste d'attente est établie : il s'agit de l'attente de biens à donner ou du contrôle à faire sur la situation des demandeurs, sur leurs conditions de vie et sur les aides éventuelles perçues d'autres associations. Une autre liste est remplie au cas par cas : elle concerne des personnes qui doivent être aidées, quand bien même elles ne sont pas insérées dans la liste. Dans ce cas, les bénéficiaires ne reçoivent souvent pas un couffin entier, mais des produits que l'association garde en surplus.

Le problème central pour l'association est d'évaluer sur le moment la réelle situation de besoin des demandeurs, et établir ainsi la priorité parmi les demandeurs. Toute l'interaction entre l'association et les demandeurs passe par la mise en récit de la vie et de la situation familiale de ces derniers. D'un côté, il s'agit de parler de ses propres " malheurs », avec des exagérations vraies ou soupçonnées par les membres de l'association ; de l'autre, il s'agit d'exiger la transparence, tout en gardant la discrétion, et d'accueillir une histoire à classer rapidement dans une catégorie afin de trouver une solution. Cette histoire peut rester dans la mémoire des responsables de l'association, qui affirment connaître tous leurs bénéficiaires et sont à leur tour souvent reconnus par le voisinage. Habib, le président de «La source de la charité de Sfax », est reconnu dans les cafés à Sfax et parfois même à Tunis ${ }^{18}$.

La pratique du récit de la vie des demandeurs d'aide crée un lien de reconnaissance, tout d'abord pour les responsables de l'association. D'ailleurs, cette pratique s'associe à une forme de contrôle social qui passe par le formatage des histoires de vie des demandeurs d'aide et de la zone d'intervention. Ceci se révèle, par exemple, dans la description stéréotypée du quartier des demandeurs, identifié avec la drogue, l'alcool, la prostitution et la délinquance. Cette forme de contrôle relève d'une double généalogie. D’abord, le contrôle peut être considéré comme le résultat de l'expérience de la clandestinité, lorsque pour établir un contact il fallait le connaître parfaitement :

C'était un travail en cachette, tout à fait différent de celui que fait aujourd'hui l'association : la discrétion, la capacité d'inventer des manières de communiquer, la capacité de connaître beaucoup de monde. Aujourd'hui on demande publiquement de l'argent, si quelqu'un a confiance il donne ; avant, quand on contactait des gens, il fallait les connaître bien, procéder petit à petit, faire des petits pas. (Entretien avec Habib, Sfax 25/05/17)

\footnotetext{
18 Observations pendant les conversations avec lui.
} 
D’ailleurs, cette forme de contrôle semble également liée aux techniques policières exercées sur les mêmes personnes qui aujourd'hui sont responsables de l'association. D’après l'un des managers associatifs qui travaille à la réforme de la charité religieuse, cette connaissance des techniques policières semble également s'appuyer sur les relais qui se sont faits dans la prison, notamment avec « les petits criminels utilisés par l'ancien parti pour "la casse" » (Entretien avec Fathi, Sfax 04/05/15).

Néanmoins, cette pratique de contrôle résulte plus simplement d'une technique administrative et bureaucratique, où l'évaluation qualitative, parfois un entretien mené par un fonctionnaire, précède normalement le classement par des critères quantitatifs et objectifs. Dans cette approche de proximité, typique des associations, la différence ne réside pas forcément dans le temps dédié aux demandeurs d'aide : les hors-liste sont de même évalués rapidement. C'est en revanche le récit lui-même qui semble assumer une valeur et un poids plus importants par rapport à la logique bureaucratique de l'administration. Par conséquent, le formalisme révèle des marges importantes de discrétion et d'arbitraire, que les associations essaient de réduire en fixant des règles bureaucratiques.

On a fait trois réunions pour décider les listes : le bureau de l'association avec les assistantes sociales, car ce sont surtout elles qui ont la maîtrise de la situation. On a fait des listes différenciées avec des priorités : d'abord les veuves, puis les familles avec des handicapés [...]. Comme on n'avait pas assez d'argent, on a essayé de prendre quelqu'un pour chaque liste. (Entretien avec Habib, Sfax 25/05/17)

Habib assure que, d'une année à l'autre, leurs critères se perfectionnent et s'affinent grâce à l'expérience accumulée : cela se révèle même nécessaire, car les demandeurs d'aide augmentent plus que les ressources de l'association.

Le cas de « La source de la charité de Sfax » montre comment, autour de la base des données des bénéficiaires du couffin de ramadan, se joue à la fois une "gouvernementalisation » des associations (Hibou, Tozy, 2015), c'est-à-dire l'adoption et la conformation de cadrages, de normes et de procédures propres de l'administration étatique, et une autonomisation personnelle des responsables des associations. Cette autonomisation ouvre certainement des marges de manœuvre arbitraires et discrétionnaires ; elle s'exprime néanmoins davantage à travers les normes et les procédures que l'association essaie de formaliser et d'affiner.

\section{Les tensions et les ambiguïtés de la mise en place des instruments du travail caritatif}

L'entrée par les instruments de l'action associative focalise l'analyse à la fois sur les ressorts multiples et sur les conséquences des processus de bureaucratisation sur les associations, en décentrant partiellement le regard des objectifs et des grands acteurs. Le livre comptable, l'organigramme et la base des données ne sont pas les seuls instruments mis en place dans le cadre de l'action associative en Tunisie : par exemple, le 
diagnostic, le projet et le rapport comme formes d'écriture codifiées sont très courants, ainsi que les dispositifs de formation, de sélection et de financement. Néanmoins, la configuration sfaxienne et la bureaucratisation des associations caritatives permettent de mieux saisir comment la redéfinition des contours du politique entraînée par les dispositifs bureaucratiques (Hibou, 2013) s'articule ultérieurement avec les dynamiques partisanes, les techniques religieuses et la gestion des quartiers et de la ville.

Loin d'être un processus univoque ayant un résultat prédéterminé, la mise en forme bureaucratique de l'action associative en Tunisie répond tout d'abord à la demande de sortir les associations de l'amateurisme, de la politisation et de la corruption. Le perfectionnement de la gestion du livre comptable montre que la remise en cause de la légitimité est le moteur premier de la bureaucratisation de l'action associative. L'accountability, la calculabilité et la comparabilité sont autant de normes acceptées pour se défendre de la discrétion, du clientélisme et des pratiques politiques autoritaires de l'État. Il faut néanmoins replacer le livre comptable dans une dynamique de plus longue durée, où l'essor postrévolutionnaire des associations lui-même est lié à la formalisation et à la légalisation des associations, contre le passé autoritaire. La légitimation par la compétence et l'appropriation de normes techniques considérées comme « neutres » entraîne une « managérialisation » du travail associatif, véritable caution de légitimité, notamment pour des associations caritatives soupçonnées de connivence avec le parti au gouvernement.

La managérialisation se lie au questionnement sur l'efficacité de l'action associative, face aux critiques d'amateurisme et d'inaction issues de l'intérieur même du milieu associatif tunisien. Les controverses et les conflits autour de l'organigramme du travail caritatif à Sfax montrent une dynamique de sectorisation du travail négociée à plusieurs niveaux. L'échec de la tentative de fusion des associations relève de l'articulation entre l'impératif d'efficacité et la quête d'autonomie des associations à la fois vis-à-vis de l'État et des plus grandes organisations. La sectorisation est une voie moyenne qui permet la résistance des petites structures associatives, la possibilité de décision de la part de leurs responsables et le maintien du dévouement et de l'enchantement des activistes (Lefèvre, 2008), ainsi que leur engagement religieux et politique. Le dévouement et l'enchantement des activistes ne sont pas en contradiction avec la redéfinition de l'activité caritative, d'un champ d'engagement religieux à une sectorisation compatible avec les politiques publiques.

À travers la construction de la base des données des bénéficiaires, la sectorisation pousse l'institutionnalisation d'une véritable bureaucratie du travail caritatif. En premier lieu, les associations semblent intégrer des relations de subsidiarité avec les autorités publiques, et notamment les collectivités locales - la municipalité et la délégation. Les associations caritatives sont appelées à collaborer avec l'État dans le cadre de l'intervention dans des zones marginalisées des quartiers, des villes et des régions. Leur capacité d'attirer des fonds des particuliers ou des organismes internationaux en fait un atout de l'action publique étatique, qui peut être sous-traitée par des associa- 
tions financièrement autonomes. La collaboration peut être directe, avec l'organisation commune d'événements, ou bien indirecte, par le passage des listes des bénéficiaires de l'aide des autorités aux associations. Il s'agit du « degré zéro » de la participation à l'action publique : les associations participent à la protection sociale en tant qu'acteurs subsidiaires, considérés mieux placés que les administrations de l'État pour répondre aux besoins de la population. Ils coordonnent la mise en œuvre d'une politique décidée ailleurs grâce à leur expérience dans le domaine.

Le cas sfaxien montre qu'en faisant partie du réseau de sociabilité islamiste, les présidents des associations caritatives, le maire et le délégué de Sfax se connaissent : les liens partisans nouent ici des relations d'action publique. Les associations semblent ainsi devenir des relais des politiques publiques de l'État, suite même à un changement partiel des administrations locales, qui voient l'entrée d'un personnel lié au gouvernement islamiste. Cette dynamique semble désamorcer la politisation des associations, tout en gardant le lien partisan redéfini par une rhétorique techniciste. De manière similaire aux pratiques analysées dans d'autres contextes d'islamisme municipal (Signoles, 2009), la prise en compte des besoins des citoyens et leur implication dans les processus de décision au local n'excluent pas le contrôle social, politique et moral. Ce contrôle s'opère notamment à travers les politiques sociales mises en œuvre par les associations et leur quadrillage du territoire.

Néanmoins, au-delà de la persistance des relations clientélistes, la base des données montre l'articulation entre une forme de gouvernement personnel et une forme de gouvernement technique. D’une part, la base des données relève du modèle de la bureaucratie d'État: afin d'atteindre l'efficacité pour soutenir et remplacer l'État, par des relations de subsidiarité, les associations s'approprient et affinent les instruments de l'État. La base des données établie entraîne des conflits entre bénéficiaires et associations, et des revendications des bénéficiaires potentiels dont l'objectif est de pouvoir rentrer dans les critères d'ayant droit à l'aide. Dans ce cadre, les responsables associatifs jouent un double jeu, en se démarquant de leur rôle de service public, tout en essayant d'affiner les critères d'attribution des aides. D'autre part, cette " gouvernementalisation de la société civile » (Hibou, Tozy, 2015 : 395) n’empêche pas la gestion très personnelle des associations révélant l'ambiguïté des normes techniques par les marges discrétionnaires des responsables des associations sur l'attribution des droits à l'aide, et donc l'émergence d'une notabilité associative.

Les instruments de l'action associative révèlent ainsi la configuration d'un mode de gouvernement hybride entre pouvoir technique et pouvoir personnel. La « décharge » (Hibou, 1999) des fonctions de l'État-providence sur des organisations institutionnalisées sous la forme souple de l'association permet justement de tenir ensemble une intervention - et la contestation des résultats de cette intervention - très personnelle et discrétionnaire des médiateurs avec l'application de techniques et outils formalisés. Formes bureaucratiques en elles-mêmes, ainsi que médiateurs, les associations se révèlent un moyen de rapprochement des instruments bureaucratiques aux activistes 
et aux bénéficiaires : elles permettent leur intervention personnelle visant moins à remettre en cause les instruments qu'à mieux les définir, tout en jouant sur les marges de manœuvre que cette définition même ouvre.

\section{Conclusion}

À travers les cas de deux associations caritatives, l'analyse s'est focalisée sur des instruments tels que le livre comptable, l'organigramme et la base des données des bénéficiaires, afin de montrer les tensions et les ambiguïtés des dynamiques de mise en forme bureaucratique de l'action associative. Les deux associations ont des trajectoires similaires d'adaptation aux normes de participation à l'action publique, notamment à l'échelle locale, en délaissant les justifications religieuses, idéologiques et politiques, et en limitant leur réseau d'interaction à une chaîne sectorielle. Cette dynamique entraîne un ensemble de changements concernant les bailleurs de fonds, les bénéficiaires et les partenaires, ainsi que l'émergence de nouvelles priorités comme l'accountability, l'efficacité et la coordination de l'action. Les ressorts et les conséquences de ces passages sont similaires : néanmoins, leur bureaucratisation est moins une transformation linéaire qu'un processus qui laisse voir des tensions et des ambiguïtés entre différents registres d'action.

Ces tensions et ambiguïtés montrent que la définition des instruments d'action associative ouvre en elle-même davantage de marges de manœuvre pour les responsables des associations. L'expression et l'exercice de ces marges de manœuvre relèvent de l'expérience personnelle, du militantisme, de la clandestinité, des ambitions et de l'idée du rôle des associations dans la nouvelle situation tunisienne. Dans ce cadre, la conformité des procédures de l'association aux normes imposées par l'État, qui lui assure la légitimité de son action, semble moins le résultat d'une réelle volonté de collaboration avec l'État qu'une appropriation des logiques étatistes de la part des responsables des associations - une « gouvernementalisation » au sein de laquelle composent différentes pratiques de contrôle de la population. En même temps, la collaboration avec l'État est bien réelle, au moins au moment où les institutions sont contrôlées par son réseau politique. Dans ce cadre, les responsables des associations jouent un rôle grandissant, en devenant à la fois des managers de la charité et les garants du fonctionnement de la chaîne sectorielle. La mise en forme bureaucratique de l'action associative se révèle ainsi un processus de passage des associations de la fonction d'intermédiation idéologique et clientéliste à une forme de gouvernement personnalisée, fondée sur les responsables-managers des associations.

\section{Bibliographie}

Allal A. (2016), « "Penser global, agir dans un bocal”. Participation locale, régulation néolibérale et situation autoritaire en Tunisie (2006-2010) », Gouvernement et action publique, vol. 2, n 2, p. 153-181. 
BAYART J.-F. (2013), « La cité bureaucratique en Afrique subsaharienne », in B. Hibou, La bureaucratisation néolibérale, Paris, La Découverte, p. 291-313.

Ben Achour S. (2011), "Société civile en Tunisie : les associations entre captation autoritaire et construction de la citoyenneté », in A. Bozzo, P.-J. LuizArd, Les sociétés civiles dans le monde musulman, Paris, La Découverte, p. 293-312.

Ben Amor R. (1994), « Politique sociale et pauvreté en Tunisie », Cahiers de la Méditerranée, vol. 49, $\mathrm{n}^{\circ} 1$, p. 189-227.

BEN NÉFISSA S. (1992), « Le mouvement associatif égyptien et l'islam : éléments d’une problématique », Monde arabe. Maghreb Machrek, n 135, p. 19-36.

De FACCI D. (2016), «L’emploi solidaire générateur d’inégalités en Tunisie ?», in M. Selim, B. CASTelLi, Réparer les inégalités ?, Paris, L’Harmattan, p. 85-101.

DE FACCI D. (2019), « Organiser l’activité caritative, revendiquer le droit au développement à Sfax. Les associations caritatives à l'épreuve de la vie citoyenne », in M. NACHI, $L a$ sociologie pragmatique et l'étude des sociétés maghrébines, Tunis, Nirvana, p. 382-405.

De Facci D., Som-1 J.-D. (2017), « La démocratie au concret : Les enjeux politiques et territoriaux de la mise en place du Budget participatif dans la Tunisie post-Ben Ali (2011-2016) », L’Année du Maghreb, nº 16, p. 245-267.

FerRé N. (2018), « La fabrique de la "société civile" entre évitement du politique et politisation : usages d'un dispositif de l'action extérieure européenne en Tunisie», Revue Gouvernance, vol. 15, n 1, p. 15-42.

FFF (2013), «Étude sur les organisations de la Société civile en Tunisie », Foundation for the Future. En ligne, consulté le 09/01/2021. URL : https://africanphilanthropy. issuelab.org/resource/study-on-civil-society-organizations-in-tunisia.html.

Halpern C., Lascoumes P., Le Galès P. (dir.) (2014), L’instrumentation de l'action publique: controverses, résistances, effets, Paris, Sciences Po Les Presses.

Hély M. (2009), Les métamorphoses du monde associatif, Paris, PUF.

Hiвоu B. (dir.) (1999), La privatisation des États, Paris, Karthala.

Hiвоu B. (2006), La force de l'obéissance. Économie politique de la répression en Tunisie, Paris, La Découverte.

Hiвоu B. (2013), « Introduction. La bureaucratisation néolibérale, ou la domination et le redéploiement de l'État dans le monde contemporain ", in B. Hiвou, La bureaucratisation néolibérale, Paris, La Découverte, p. 7-20.

Hiвou B., Tozy M. (2015), « Gouvernement personnel et gouvernement institutionnalisé de la charité: L'INDH au Maroc », in I. Bono, B. Hibou, H. Meddeb, M. Tozy, L'État d'injustice au Maghreb. Maroc et Tunisie, Paris, Karthala, p. 379-428.

Laroussi H. (2009), Micro-crédit et lien social en Tunisie : la solidarité instituée, Paris, Karthala. 
Lascoumes P., Le Galès P. (2004), Gouverner par les instruments, Paris, Sciences Po Les Presses.

Laville J.-L., Salmon A. (2015), Associations et Action publique : Solidarité et société, Paris, Desclée de Brouwer.

LefÈVRE S. (2008), « Mobiliser les gens, mobiliser l'argent : les ONG au prisme du modèle entrepreneurial », thèse de doctorat de science politique, Paris, Université Paris-1 «Panthéon-Sorbonne ».

Merone F., Soli E. (2013), « Tunisia: the Islamic associative system as a social counterpower », Opendemocracy.net. En ligne, consulté le 31/10/2020. URL: https:/www. opendemocracy.net/north-africa-west-asia/evie-soli-fabio-merone/tunisia-islamicassociative-system-as-social-counter-power.

Merone F., Sigillò E., De Facci D. (2018), « Nahda and Tunisian Islamic Activism », in D. Conduit, S. Akbarzadeh, New Opposition in the Middle East, Singapore, Palgrave Macmillan, p. 177-201.

PNud (2015), Les priorités du développement en Tunisie vues par la société civile. En ligne, consulté le 11 février 2021. URL : https://jamaity.org/publication/les-priorites-dudeveloppement-en-tunisie-vues-par-la-societe-civile/.

Redissi H. (2007), « Tunisie : État fort, société civile faible », Maghreb-Machrek, n 192, p. 89-117.

Sigillò E. (2016), « Mobilizing for or through development? Trajectories of civic activism in post-authoritarian Tunisia », thèse de doctorat de science politique, Florence, Scuola Normale Superiore de Firenze.

Sigillò E. (2020a), "Ennahdha et l'essor des associations islamiques en Tunisie : revendiquer l'islam politique au-delà de la dimension partisane ? », L'Année du Maghreb, n² 22, p. 113-129.

Sigiliò E. (2020b), « Se spécialiser dans le secteur associatif : la transformation de la charité islamique à l'aune de la reconfiguration du jeu politique en Tunisie ", in L. Ruiz De Elvira, S. SaEIdNia, Les mondes de la bienfaisance. Une approche comparative des pratiques $d u$ « bien », Paris, CNRS éditions.

Signoles A. (2009), « L'islamisme municipal, enjeu et garant de la modernisation des pratiques politiques? », Critique internationale, $\mathrm{n}^{\circ}$ 1, p. 9-19.

Simonet M. (2010), Le travail bénévole. Engagement citoyen ou travail gratuit ?, Paris, La Dispute. 